\title{
Sumoylation of Drosophila Transcription Factor STAT92E
}

\author{
Juha Grönholm a Daniela Ungureanu ${ }^{a}$ Sari Vanhatupa ${ }^{a}$ Mika Rämet $^{a, b}$ \\ Olli Silvennoinen ${ }^{a, c}$ \\ ${ }^{a}$ Institute of Medical Technology, University of Tampere, and bepartment of Pediatrics, and \\ ${ }^{c}$ Center for Laboratory Medicine, Tampere University Hospital, Tampere, Finland
}

\section{Key Words}

Signal transducer and activator of transcription - STAT •

Small ubiquitin-like modifier · Sumo $\cdot$ Sumoylation •

Post-translational modification

\begin{abstract}
STAT92E is an essential transcription factor in Drosophila melanogaster for the development of several organs and the immune system. The JAK/STAT pathway employs different evolutionary conserved regulatory mechanisms to control biological processes. Numerous transcription factors in both mammals and invertebrates have been shown to be either activated or inhibited by a covalent modification with a small ubiquitin-like modifier (Sumo). Here, we show that Drosophila STAT92E is modified by Sumo at a single lysine residue 187 in S2 cells. Mutation of Lys187 increases the transcriptional activity of STAT92E, thus suggesting that sumoylation of STAT92E has a repressive role in the regulation of the JAK/ STAT pathway in Drosophila melanogaster.
\end{abstract}

Copyright $\odot 2010$ S. Karger AG, Basel
(C) 2010 S. Karger AG, Basel

$1662-811 X / 10 / 0026-0618 \$ 26.00 / 0$

Fax +4161306 1234

E-Mail karger@karger.ch

www.karger.com
Accessible online at:

www.karger.com/jin

\section{Introduction}

The JAK/STAT pathway is highly conserved in the evolution from invertebrates to humans. The JAK/STAT signaling cascade is essential for several biological processes, including the control of hematopoiesis and immune responses, as well as for cellular homeostasis and embryonic development $[1,2]$. The human JAK/STAT pathway consists of 7 STATs and 4 JAKs [3]. In Drosophila melanogaster, the pathway employs only a single Janus kinase Hopscotch (Hop) and the transcription factor STAT92E as well as the receptor Domeless (Dome) [2], thus making Drosophila a useful non-redundant model to study this pathway.

The activation mechanism of the JAK/STAT pathway is shared by invertebrates and humans. In Drosophila, 3 secreted ligands (Unpaired 1-3, Upd1-3) [4-6] induce the homodimerization of the Dome receptors allowing the Hop kinases to phosphorylate tyrosine residues in the receptors, thus creating docking sites for the $\mathrm{SH} 2$ domain of STAT92E. Following the interaction with Dome, STAT92E becomes phosphorylated by Hop, leading to the homodimerization and nuclear translocation of STAT92E. In the nucleus, STAT92E binds to its target DNA sequences and acts as activator of transcription for

Prof. Olli Silvennoinen

Institute of Medical Technology

University of Tampere

FI-33014 Tampere (Finland)

Tel. +358 33551 7845, Fax +358 33551 7332, E-Mail olli.silvennoinen@ uta.fi 
several target genes such as Tep 2 and Turandot stress genes $[1,7]$.

Cellular homeostasis requires tight regulation of the JAK/STAT signaling cascade, and deregulation or disturbances of the pathway are associated with or cause several human diseases such as myeloproliferative diseases, cancer, immune deficiencies, autoimmunity and allergy [8-10]. In Drosophila, deregulation of the JAK/ STAT pathway causes overproliferation of plasmatocytes, tumor formation and developmental abnormalities $[2,11]$. The basic regulatory mechanisms of the JAK/ STAT pathway appear to be well conserved between Drosophila and mammals. Previously known negative regulators of the Drosophila JAK/STAT pathway include a family of SOCS-like Drosophila genes, dPIAS, also known as Zimp or Su(var)2-10, Ptp61F (protein-tyrosine phosphatase $61 \mathrm{~F}$ ) and an N-terminally truncated form of STAT92E ( $\Delta$ NSTAT92E) [12-15]. Transcriptional activity of STAT92E is inhibited by direct interaction with dPIAS, and the correct dPIAS/STAT92E ratio is crucial for normal hematopoiesis and organogenesis in Drosophila [13]. PIAS1 was initially shown to interfere with the promoter-binding activity of tyrosine-phosphorylated STAT1 in mammals [16]. PIAS1 functions also as an E3 ligase for small ubiquitin-like modifier (Sumo), and STAT1 was identified as a substrate for PIAS1-induced sumoylation. Sumoylation of STAT1 has an inhibitory effect on STAT1-mediated transcriptional activation [17, $18]$.

Several transcription factors in mammals as well as in Drosophila are regulated by sumoylation. Sumoylation is a reversible post-translational modification in which a Sumo moiety is covalently conjugated to the lysine residues on target proteins through a process analogous to ubiquitination. The sumoylation pathway is conserved in all eukaryotic organisms and consists of E1-activating enzymes, E2-conjugating enzymes and E3 ligases as well as of the family of the Sumo cleaving proteases $[19,20]$. The Sumo conjugation pathway is less redundant in invertebrates; for example, in vertebrates, 4 Sumo variants (Sumo 1-4) are present, while Drosophila expresses only 1 Sumo ortholog known as Smt3 [19]. Sumoylation can affect target proteins by multiple mechanisms, altering their subcellular localization, molecular interactions, stability or the enzymatic activity of the proteins [20]. At the transcriptional regulation level, sumoylation is mostly associated with the repression of gene expression. The mechanism of Sumo-mediated transcriptional inhibition is often associated with the recruitment of various chromatin-modifying or-associated proteins or protein com- plexes [21]. For example, the transcriptional activity of Drosophila Sp3 is inhibited by sumoylation-mediated recruitment of a repression complex formed by MEP-1, Mi-2 and Sfmbt [22]. This study aims to investigate whether sumoylation is involved in the regulation of the JAK/STAT pathway in Drosophila.

\section{Methods}

Antibodies and Reagents

The following antibodies were used: anti-Flag M2 (SigmaAldrich); anti-influenza hemaglutinin (HA) epitope antibody (Covance); anti-Drosophila STAT92E-N-terminus (dN-17; Santa Cruz); biotinylated anti-mouse (DakoCytomation, Glostrup, Denmark) and biotinylated anti-goat (DakoCytomation). Also, streptavidin-biotin horseradish peroxidase conjugate (GEHealthcare), protein $\mathrm{G}$ sepharose (GE-Healthcare) and N-ethylmaleimide (Sigma-Aldrich) were used.

Plasmid Constructs

STAT92E (clone RE14194) was obtained from the Drosophila Genomics Resource Center (Indiana University, Bloomington, Ind., USA) and amplified by PCR using $5^{\prime}$-primer containing Notl site and $3^{\prime}$-primer containing Flag epitope tag and Pmel restriction site. STAT92E was cloned into a pMT/V5-HisA expression vector (Invitrogen). PCR-amplified C-terminally Flagtagged STAT92E was also cloned into Not1 and Kpn1 sites of the pcDNA3.1(-) expression vector. The pMT-STAT92E K187R-Flag was created by PCR mutagenesis with the following primers: $5^{\prime}$ GGTATGGTCACACCCAGGGTGGAHCTGTACGAG-3' and 5'-CTCGTACAGCTCCACCCTGGGTGTGACCATACC-3'. Sumo-1-Flag and Sumo-1-Flag-Flag were a kind gift from Dr. $\mathrm{H}$. Yasuda. Senp1-Flag, Senp1 C603S, Sumo-1 GA-His and Sumo-1His as well as Sumo-3-HA were provided by Prof. J. Palvimo, Turandot $M$-luciferase (TotM-luc), Actin 5C- $\beta$-galactosidase reporters and Hop-Tum-l were provided by Prof. J.-L. Imler. Smt3-HA plasmid was a kind gift from Dr. A.J. Courey. 10xSTAT-luciferase reporter plasmid was a kind gift from Dr. E. Bach, and pMT-Upd plasmid was kindly provided by Dr. Michael Boutros.

\section{Cell Culture, Transfection and Immunodetection}

Cos- 7 cells were cultured at $37^{\circ} \mathrm{C}$ in Dulbecco's modified Eagle's medium supplemented with $10 \%$ ( $\mathrm{vol} / \mathrm{vol}$ ) fetal bovine serum, $100 \mathrm{U} / \mathrm{ml}$ penicillin and $50 \mathrm{mg} / \mathrm{ml}$ streptomycin. S2 cells were cultured at $25^{\circ} \mathrm{C}$ in Schneider medium (Sigma-Aldrich) supplemented with $10 \%$ (vol/vol) FBS, $100 \mathrm{U} / \mathrm{ml}$ penicillin and $50 \mathrm{mg} /$ $\mathrm{ml}$ streptomycin. Cos-7 cells $\left(5 \times 10^{6}\right)$ were transfected by electroporation with a total amount of $3.0 \mu \mathrm{g}$ plasmid DNA using Bio-Rad gene-pulser at $260 \mathrm{~V}$ and $960 \mu \mathrm{F}$. Cells were lysed in 1\% Triton X lysis buffer containing $5 \mathrm{mM} \mathrm{N}$-ethylmaleimide [17]. One milligram of protein from total cell lysates was subjected to immunoprecipitation, SDS-PAGE electrophoresis and Western blotting as previously described [17]. Approximately $1.5 \times 10^{7} \mathrm{~S} 2$ cells were transfected with a total amount of $10 \mu \mathrm{g}$ DNA using polyethyleneimine [23]. Two hours after transfection, the medium was changed to a fresh medium containing $10 \mu \mathrm{g}$ of STAT92E 3'-UTR dsRNA to inhibit the expression of endogenous STAT92E, and 


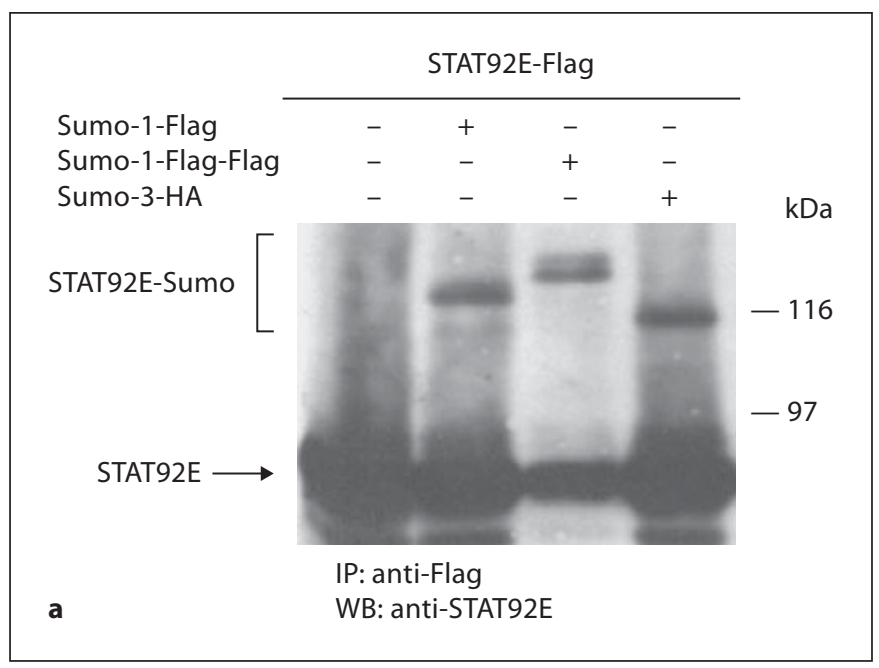

Fig. 1. STAT92E is sumoylated in Cos-7 cells. a Sumoylation of STAT92E in Cos-7 cells by human Sumo-1 and human Sumo-3. Cos-7 cells were transiently transfected with STAT92E-Flag with or without different Sumo constructs as indicated. Cells were lysed $48 \mathrm{~h}$ after transfection. Equal amounts of lysates were immunoprecipitated by using anti-Flag antibody, followed by SDS-PAGE and immunoblotting with anti-STAT92E antibody.

$500 \mu \mathrm{M} \mathrm{CuSO}$ to induce the expression of the pMT vector. The cells were lysed after $72 \mathrm{~h}$ and immunoprecipitations were carried out as described [17].

\section{RNA Interference}

For RNA interference (RNAi) assay with endogenous STAT92E, double-stranded DNA was synthesized from the cDNA of the 3'-UTR of STAT92E using 2-step PCR with nested primers, the second primers containing a T7 promoter sequence at the $5^{\prime}$ end. STAT92E 3'-UTR dsRNA was synthesized from the product of the second PCR with the T7 MegaScript RNA polymerase (Ambion), according to the manufacturer's instructions. dPIAS and GFP dsRNAs were created as above, but the PCR primers were designed in the middle of the protein coding sequence of the genes. dsRNAs were added to $\mathrm{S} 2$ culture medium $72 \mathrm{~h}$ prior to cell lysis.

\section{Luciferase Assay}

S2 cells $\left(1.0 \times 10^{6}\right)$ were transfected with $0.1 \mu \mathrm{g}$ TotM-luc reporter plasmid or with $0.001 \mu \mathrm{g} 10 x$ STAT-luciferase reporter plasmid simultaneously with $0.1 \mu \mathrm{g}$ Actin $5 C$ - $\beta$-galactosidase, with or without $0.1 \mu \mathrm{g}$ pMT-Upd and with either $0.2 \mu \mathrm{g}$ empty pMT vector, pMT-STAT92E or pMT-STAT92E K187R using Fugene 6 transfection reagent, according to the manufacturer's instructions (Roche). $\mathrm{CuSO}_{4}(500 \mu \mathrm{M})$ was added to the medium $24 \mathrm{~h}$ after transfection to induce the expression of the pMT vector. Cells were lysed after $72 \mathrm{~h}$ with reporter lysis buffer (Promega), according to the manufacturer's instructions. The luciferase values were normalized against $\beta$-galactosidase values.

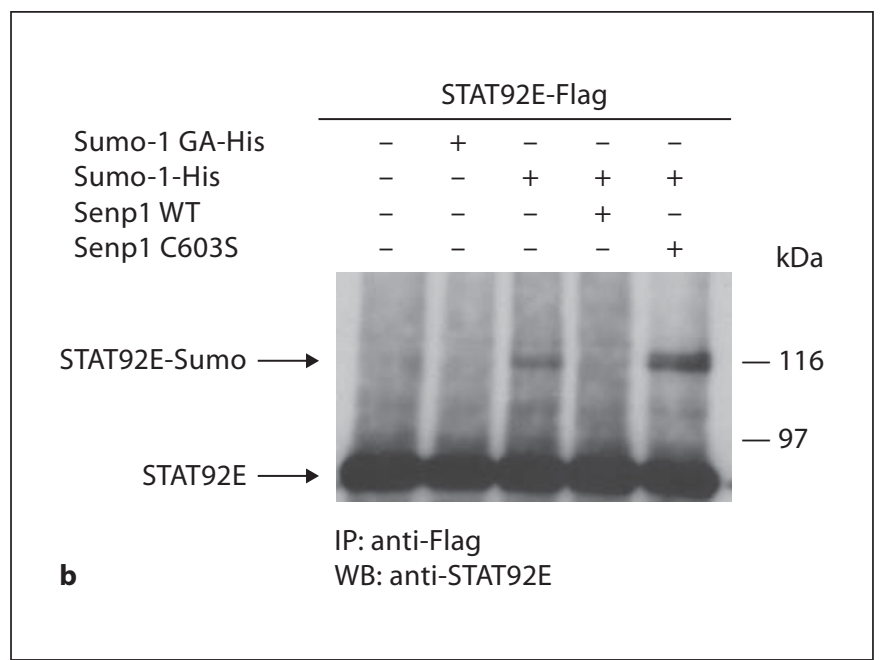

b Senp1 desumoylates STAT92E. Cos-7 cells were transfected with STAT92E-Flag together with Sumo-1-His or Sumo-1 GA-His as indicated and with Senp1 or with catalytically inactive Senp1 C603S. Cell lysis, immunoprecipitations and immunoblotting were carried out as described above. IP = Immunoprecipitation; $\mathrm{WB}=$ Western blot.

\section{Results}

\section{STAT92E Is Modified by Sumo in Mammalian Cells}

In order to investigate if Drosophila STAT92E is a target for sumoylation, the initial experiments were performed in mammalian Cos-7 cells with well-defined mammalian components of the sumoylation pathway. Cells were transfected with Flag-tagged STAT92E alone or together with Flag-tagged human Sumo-1, or tandem Flag-tagged human Sumo-1, or HA-tagged human Sumo3. Cell lysates were immunoprecipitated with anti-Flag antibody, and anti-Drosophila STAT92E-N-terminal antibody was used in immunoblotting. Anti-STAT92E antibody detected protein bands corresponding to the molecular mass of STAT92E (fig. 1a). In addition, slower migrating bands were detected when STAT92E was expressed together with Sumo-1 or Sumo-3, indicating that these bands are STAT92E-Sumo-1/3 conjugation complexes. The band shift of the slower migrating band with tandem Flag-tagged Sumo-1 compared with single Flag-tagged Sumo-1 corresponds to the size of a Flag-tag, showing that the upper bands are STAT92E-Sumo-1 bands. The STAT92E-Sumo-1/3 complex is a branched chain molecule, and thus, its migration in the SDS-PAGE gel is abnormal, creating bands of approximately $116 \mathrm{kDa}$. 
Fig. 2. STAT92E is sumoylated at lys187. a Schematic domain structure of STAT92E based on sequence comparison with mammalian STATs. Amino acid sequence of the Sumo consensus site in the coiled coil domain is indicated. The Sumo consensus sequence is not complete in the C-terminal site, although it resembles the human STAT1 sumoylation site (Tyr711 is the critical phosphorylation site in the activated STAT92E). b The K187R mutation abrogates sumoylation of STAT92E. S2 were transiently transfected with STAT92E WT-Flag or STAT92E K187R-Flag with or without Smt3-HA as indicated. Two hours after transfection, $10 \mu \mathrm{g}$ of STAT92E 3'UTR dsRNA was added to the medium to inhibit the expression of the endogenous STAT92E. Equal amounts of lysates were immunoprecipitated by using anti-Flag or anti-STAT92E-N-terminal antibodies following SDS-PAGE and immunoblotting with anti-STAT92E-N-terminal and antiHA antibodies. c Effect of RNA interference on endogenous STAT92E levels in S2 cells by double-stranded RNA targeted on STAT92E 3'-UTR. dsRNA was added to cells $2 \mathrm{~h}$ after DNA transfection. Unspecific bands are marked with an asterisk. IP = Immunoprecipitation; WB = Western blot.
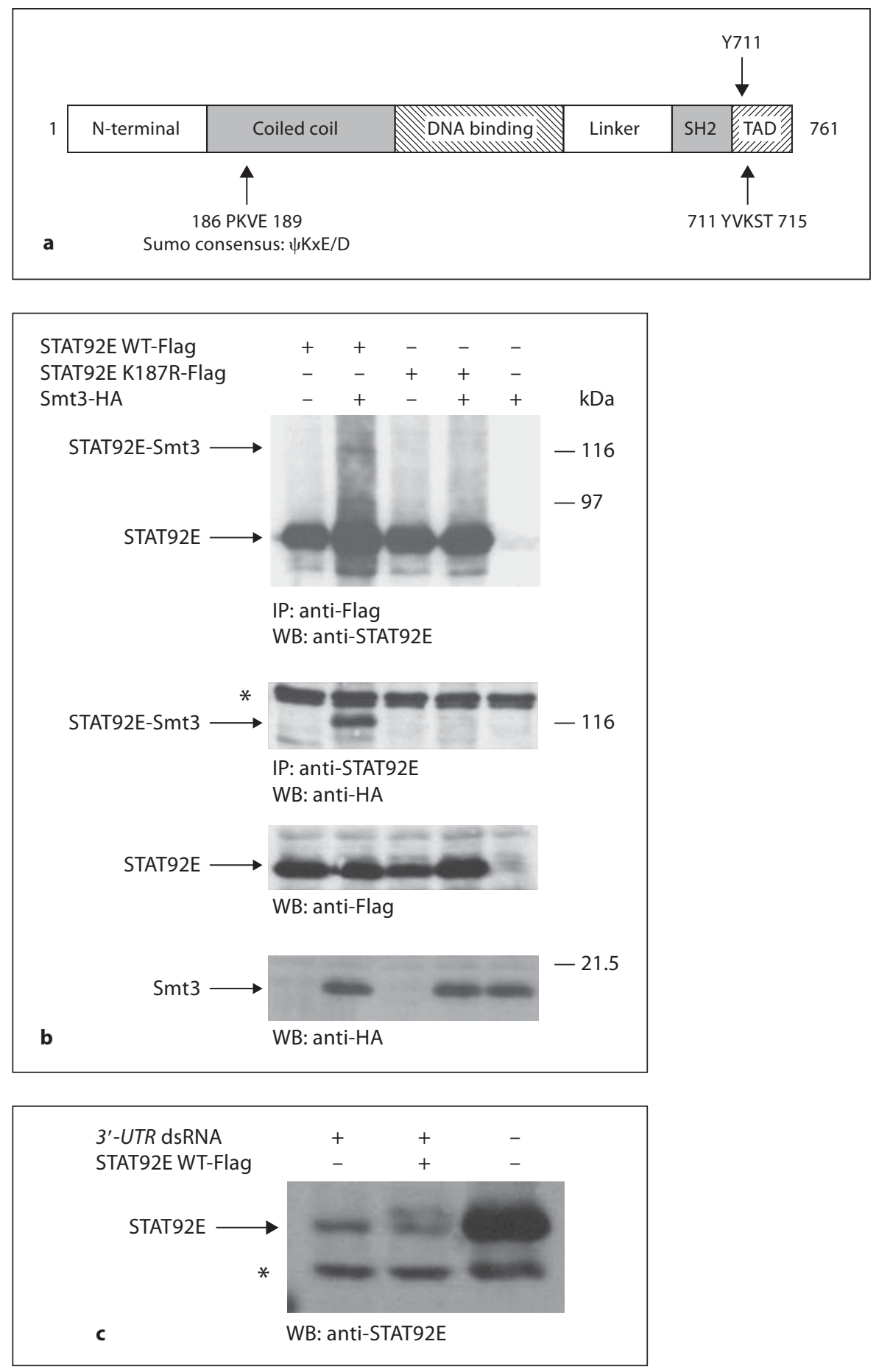

To obtain further proof that the slower migrating STAT92E proteins are sumoylated, we investigated if the Sumo-specific protease Senpl could remove Sumo molecules from STAT92E and subsequently eliminate the slower migrating bands. Cos-7 cells were transfected with
STAT92E and His-tagged Sumo-1 constructs with or without Senp1 expression constructs. A catalytically inactive mutant Senp1-C603S and a conjugation-deficient mutant of Sumo-1 (Sumo-1 GA-His) were used as controls [24]. As shown in figure 1b, expression of Senp1 
abolished the protein band corresponding to Sumo-1 conjugation of STAT92E, while the expression of Senp1C603S slightly increased the amount of sumoylated STAT92E. The Sumo-1 GA-His mutant was not attached to STAT92E, thus confirming the specificity of the conjugation reaction. Sumo-1-His and Sumo-1 GA-His expression levels were detected (data not shown). These results suggest that Drosophila STAT92E can be sumoylated in cells.

\section{STAT92E Is Sumoylated in Drosophila S2 Cells on Lysine 187}

Next, we wanted to study if STAT92E is sumoylated also in a more physiological environment and Drosophila S2 cells were used. Sumo is conjugated to a lysine residue in target proteins most often within a consensus sequence site, $\Psi \mathrm{KxE} / \mathrm{D}$, where $\Psi$ is a large hydrophobic amino acid [20]. The protein sequence analysis of STAT92E displays a single putative sumoylation site between amino acids 186-189 (PKVE) (fig. 2a). To investigate if this is a Sumobinding site in STAT92E, Lys187 was mutated to arginine. Flag-tagged STAT92E WT and Flag-tagged STAT92E K187R were transfected into Drosophila S2 cells with or without HA-tagged Smt3. The expression of the endogenous STAT92E protein was inhibited using RNAi with STAT92E 3'-UTR dsRNA (fig. 2c). S2 cell lysates were im-

Fig. 3. K187R mutation increases STAT92E transcriptional activity. a S2 cells were transiently transfected with the pMT-V5-HisA vector, STAT92E WT-Flag or STAT92E K187R-Flag together with TotM-luc reporter and Actin 5C- $\beta$-galactosidase with or without Upd to activate the JAK/STAT pathway. Twenty-four hours after transfection, $500 \mu \mathrm{M} \mathrm{CuSO}_{4}$ was added to the medium to induce expression of STAT92E and Upd from the pMT vectors. Seventytwo hours after transfection, the cells were lysed with reporter lysis buffer and luciferase activities were measured and normalized against $\beta$-galactosidase activities. The relative TotM-luc activity of Upd-induced STAT92E WT was set as one. Equal amounts of lysates were subjected to SDS-PAGE following immunoblotting with anti-Flag antibody in order to detect the expression of transfected STAT92E WT and STAT92E K187R. b K187R mutation increases STAT92E transcriptional activity on 10xSTAT-luc reporter. Transfections and lysis were carried out as in a. The relative 10xSTAT-luc activity of Upd-induced STAT92E WT was set as one. Immunoblotts for cell lysates were carried out as described above. c dPIAS RNAi increases TotM-luc activity. S2 cells were transiently transfected with TotM-luc reporter and Actin $5 C$ - $\beta$ galactosidase together with indicated dsRNAs and with or without constitutively active Hopscotch (Hop-Tum-l) to activate the JAK/STAT pathway. Cells were lysed $72 \mathrm{~h}$ after transfection. Error bars indicate SEM, calculated from 3 independent transfections. $\mathrm{WB}=$ Western blot.
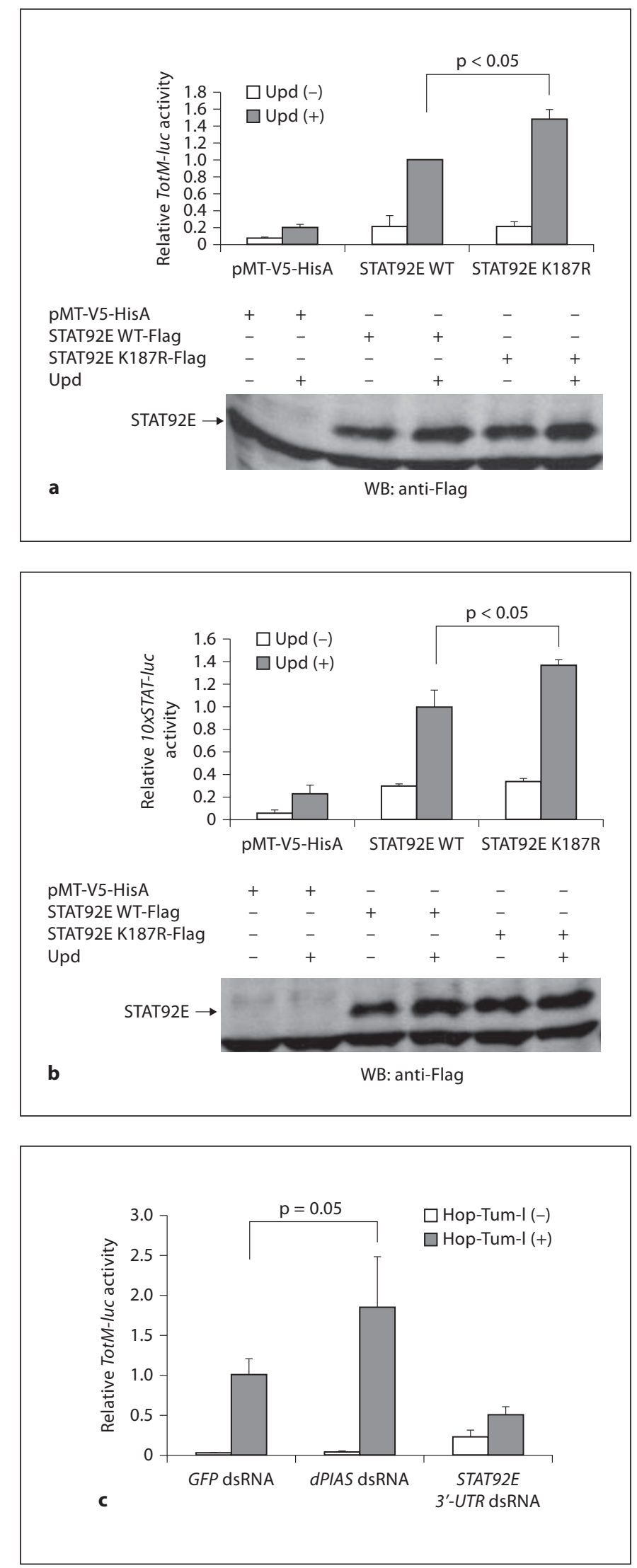
munoprecipitated with anti-Flag and anti-STAT92E antibodies followed by Western blotting with anti-STAT92E and anti-HA antibodies. As shown in figure $2 \mathrm{~b}$, the K187R mutation abrogated the modification by Smt3, indicating that Lys187 is the only sumoylation site in STAT92E.

\section{K187R Mutation Increases STAT92E Transcriptional} Activity

Sumoylation is known to regulate the activity of several transcription factors, including human STAT1. Next, we wanted to study if sumoylation affects the transcriptional activity of STAT92E. The transcriptional activities of STAT92E WT and the sumoylation-deficient STAT92E K187R were analyzed in S2 cells using a reporter gene driven by the STAT92E-inducible stress gene Turandot $M$ promoter. Expression of the Dome ligand Upd resulted in a 3-fold induction of transcription with endogenous JAK/STAT proteins. In the absence of Upd, the expression of both STAT92E WT and K187R induced transcription at similar levels, but upon Upd stimulation, the sumoylation-deficient STAT92E mutant K187R showed a significantly higher level of transcriptional activity (fig. 3a). Equal amounts of lysates were subjected to SDS-PAGE following immunoblotting with anti-Flag antibody to show that STAT92E WT and STAT92E K187R were expressed equally (fig. 3a, lower panel). To verify this observation, we used a reporter gene driven by a different STAT92E responsive element from the SOCS36E enhancer region. Figure $3 \mathrm{~b}$ shows that the STAT92E K187R mutant had an increased transcriptional activity compared with STAT92E WT after Upd stimulation with the 10xSTAT92E-luc reporter gene as well. Taken together, these results suggest that sumoylation has an inhibitory effect on STAT92E-induced gene expression.

\section{Discussion}

Sumoylation is a common posttranslational modification for transcription factors, which in most cases has an inhibitory effect on transcription. In this study, we demonstrate that STAT92E is subject to Sumo modification and that sumoylation appears to play a negative regulatory role in the Drosophila JAK/STAT pathway.

Sumoylation has been challenging to study due to the highly dynamic and reversible nature of the modification resulting in low cellular levels of Sumo-modified proteins. Also, the Sumo-modified fraction of STAT92E was notably smaller than the unmodified STAT92E, and overexpression of STAT92E and Sumo was needed to detect the Sumo-modified STAT92E. The results with Senp1 coexpression are in line with the specificity of Sumo conjugation. Sumo is deconjugated very rapidly by Sumo-specific proteases, and expression of the inactive Senp1-C603S resulted in increased levels of STAT92E sumoylation, possibly due to competitive inhibition of endogenous Senps.

STAT92E was found to become sumoylated at Lys187. Human STAT1 is sumoylated at Lys703, which is localized in the C-terminus close to the phosphorylated activating Tyr701 residue [17]. Drosophila STAT92E has a lysine in a similar position (Tyr711-Lys713), but mutation of this lysine did not alter the amount of sumoylation when compared with STAT92E WT (data not shown). Thus, Lys187 appears to be the only sumoylation site in STAT92E. This lysine is not conserved in mammalian STATs, but there is a putative Sumo consensus site found at least in the coiled coil domain of human STAT2 (Lys234). Sumoylation of human STAT2 has not been reported.

Mutation of Lys187 resulted in increased transcriptional activity of STAT92E, suggesting that sumoylation has an inhibitory effect on STAT92E. Removal of endogenous Sumo E3 ligase dPIAS by dsRNA increased the STAT92E activity on TotM promoter approximately to the same level as K187R mutation in STAT92E (fig. 3c), suggesting that sumoylation of STAT92E is involved in dPIAS-mediated inhibition of STAT92E.

The mechanisms of how sumoylation is affecting STAT92E are presently unknown, but several possible mechanisms can be envisioned. The sumoylation site Lys187 is localized in the coiled coil domain, which in the mammalian system is involved in nuclear transport of STATs. The coiled coil domain of STATs is composed of $4 \alpha$-helixes that are pointing out from the DNA-bound STAT dimer, forming a hydrophilic surface able to interact with other molecules [9]. Thus, sumoylation of Lys187 may interrupt the interaction between STAT92E and its transcriptional coregulators or the proteins involved in its nuclear translocation. Alternatively, sumoylation may lead to the recruitment of histone deacetylases to the promoter or allow the interaction with a transcription repression complex similarly to Drosophila Sp3 [22]. The effect of sumoylation on DNA-binding properties of STAT92E was not analyzed, but the coiled coil domain is not contacting DNA, suggesting that direct effects upon the promoter-binding activity are less likely. 
In the mammalian system, sumoylation has been implicated in the regulation of interferon-induced gene responses. STAT1 and other coregulatory proteins have been shown to become sumoylated [25], but the physiological role of this modification remains to be revealed. The sumoylation pathway is well conserved during evolution and the lower redundancy of the Drosophila system provides a useful system for functional and mechanistic analysis of the pathway. Our findings that STAT92E is subject to Sumo modification imply that the Drosophila system can now be utilized to investigate the physiological function of sumoylation in JAK/STAT signaling.

\section{Acknowledgements}

We thank Paula Kosonen, Merja Lehtinen, Leena Mäkinen and Tuula Myllymäki for their excellent technical assistance. Also, PhD Juha Saarikettu is acknowledged for the critical comments on the manuscript.

M.R. was supported by grants from the Academy of Finland, the Foundation for Pediatric Research, the Sigrid Juselius Foundation and the Emil Aaltonen Foundation, and from the Competitive Research Funding of the Pirkanmaa Hospital District. O.S. was supported by the Medical Research Council of Academy of Finland, the Sigrid Juselius Foundation, the Medical Research Fund of Tampere University Hospital, the Finnish Cancer Foundation and the Tampere Tuberculosis Foundation.

\section{References}

$>1$ Hombría JC, Brown S: The fertile field of Drosophila Jak/STAT signalling. Curr Biol 2002;12:R569-R575.

$\checkmark 2$ Agaisse H, Perrimon N: The roles of JAK/ STAT signaling in Drosophila immune responses. Immunol Rev 2004;198:72-82.

$\checkmark 3$ Darnell JE Jr: STATs and gene regulation. Science 1997;277:1630-1635.

4 Harrison DA, McCoon PE, Binari R, Gilman $\mathrm{M}$, Perrimon N: Drosophila unpaired encodes a secreted protein that activates the JAK signaling pathway. Genes Dev 1998;12 3252-3263.

5 Castelli-Gair Hombría J, Brown S, Häder S, Zeidler MP: Characterisation of Upd2, a Drosophila JAK/STAT pathway ligand. Dev Biol 2005;288:420-433.

6 Agaisse H, Petersen UM, Boutros M, MatheyPrevot B, Perrimon N: Signaling role of hemocytes in Drosophila JAK/STAT-dependent response to septic injury. Dev Cell 2003; 5:441-450.

7 Lemaitre B, Hoffmann J: The host defense of Drosophila melanogaster. Annu Rev Immunol 2007;25:697-743.

$>8$ Zhao R, Xing S, Li Z, Fu X, Li Q, Krantz SB, Zhao ZJ: Identification of an acquired JAK2 mutation in polycythemia vera. J Biol Chem 2005;280:22788-22792.

$\checkmark 9$ Schindler CW: Series introduction. JAKSTAT signaling in human disease. J Clin Invest 2002;109:1133-1137.

>10 Imada K, Leonard WJ: The Jak-STAT pathway. Mol Immunol 2000;37:1-11.
-11 Luo H, Rose P, Barber D, Hanratty WP, Lee S, Roberts TM, D’Andrea AD, Dearolf CR: Mutation in the Jak kinase JH2 domain hyperactivates Drosophila and mammalian Jak-Stat pathways. Mol Cell Biol 1997; 17: 1562-1571.

12 Callus BA, Mathey-Prevot B: SOCS36E, a novel Drosophila SOCS protein, suppresses JAK/STAT and EGF-R signalling in the imaginal wing disc. Oncogene 2002;21:48124821.

13 Betz A, Lampen N, Martinek S, Young MW, Darnell JE Jr: A Drosophila PIAS homologue negatively regulates stat92E. Proc Natl Acad Sci USA 2001;98:9563-9568.

-14 Müller P, Kuttenkeuler D, Gesellchen V, Zeidler MP, Boutros M: Identification of JAK/STAT signalling components by genome-wide RNA interference. Nature 2005; 436:871-875.

15 Henriksen MA, Betz A, Fuccillo MV, Darnell JE Jr: Negative regulation of STAT92E by an N-terminally truncated STAT protein derived from an alternative promoter site. Genes Dev 2002;16:2379-2389.

16 Liu B, Liao J, Rao X, Kushner SA, Chung CD, Chang DD, Shuai K: Inhibition of Stat1-mediated gene activation by PIAS1. Proc Natl Acad Sci USA;95:10626-10631.

17 Ungureanu D, Vanhatupa S, Kotaja N, Yang J, Aittomaki S, Jänne OA, Palvimo JJ, Silvennoinen O: PIAS proteins promote SUMO-1 conjugation to STAT1. Blood 2003;102:33113313 .
18 Ungureanu D, Vanhatupa S, Grönholm J, Palvimo JJ, Silvennoinen O: SUMO-1 conjugation selectively modulates STAT1-mediated gene responses. Blood 2005;106:224-226.

19 Talamillo A, Sánchez J, Barrio R: Functional analysis of the SUMOylation pathway in Drosophila. Biochem Soc Trans 2008;36: 868-873

20 Hay RT: SUMO: a history of modification. Moll Cell 2005;18:1-12.

$>21$ Garcia-Dominguez M, Reyes JC: SUMO association with repressor complexes, emerging routes for transcriptional control. Biochim Biophys Acta 2009; 1789:451-459.

-22 Stielow B, Sapetschnig A, Krüger I, Kunert N, Brehm A, Boutros M, Suske G: Identification of SUMO-dependent chromatin-associated transcriptional repression components by a genome-wide RNAi screen. Mol Cell 2008;29:742-754

23 Boussif O, Lezoualc'h F, Zanta MA, Mergny MD, Scherman D, Demeneix B, Behr JP: A versatile vector for gene and oligonucleotide transfer into cells in culture and in vivo: polyethylenimine. Proc Natl Acad Sci USA 1995;92:7297-7301.

$24 \mathrm{Xu}$ Z, Chau SF, Lam KH, Chan HY, Ng TB, $\mathrm{Au}$ SW: Crystal structure of the SENP1 mutant C603S-SUMO complex reveals the hydrolytic mechanism of SUMO-specific protease. Biochem J 2006;398:345-352.

25 Lee JH, Park SM, Kim OS, Lee CS, Woo JH, Park SJ, Joe EH, Jou I: Differential SUMOylation of LXRalpha and LXRbeta mediates transrepression of STAT1 inflammatory signaling in IFN-gamma-stimulated brain astrocytes. Mol Cell 2009;35:806-817. 